\title{
Geometric Mechanics Based Nonlinear Model Predictive Spacecraft Attitude Control with Reaction Wheels
}

\author{
Dae Young Lee* \\ University of Texas, Austin, Texas 78759 \\ Rohit Gupta \\ University of Michigan, Ann Arbor, Michigan 48109 \\ Uroš V. Kalabićc \\ Mitsubishi Electric Research Laboratories, Cambridge, Massachusetts 02139 \\ and \\ Anthony M. Bloch, I James W. Cutler,: $\stackrel{\text { ** }}{-}$ and Ilya V. Kolmanovsky立 \\ University of Michigan, Ann Arbor, Michigan 48109 \\ DOI: $10.2514 / 1 . G 001923$
}

\begin{abstract}
This paper develops a nonlinear model predictive controller for constrained attitude maneuvering of a fully actuated spacecraft with reaction wheels. In the proposed control algorithm, a Lie group variational integrator is used as a predictive model. The nonlinear model predictive control problem is formulated in the form of a discrete-time optimal control problem over each prediction horizon, and a numerical solver is used to solve the necessary conditions for optimality for this discrete-time optimal control problem. The numerical solver uses the indirect single shooting method. The control constraints and exclusion zone constraints are handled using the exterior penalty function approach. Simulation results are presented and compared with the case of a fully actuated spacecraft without reaction wheels. The nonlinear model predictive controller is shown to provide a large domain of attraction.
\end{abstract}

\section{Nomenclature}

$(B)_{A} \quad=1 / 2\left(B-B^{T}\right) ;$ antisymmetric part of square matrix $B$

$(B)_{S} \quad=1 / 2\left(B+B^{T}\right)$; symmetric part of square matrix $B$

$\|B\|_{F}=\sqrt{\sum_{i=1}^{n} \sum_{j=1}^{m}\left|B_{i, j}\right|^{2}} ;$ Frobenius norm of $n \times m$ matrix $B$

$D_{x} \mathcal{F}=$ functional or variational derivative of the differentiable scalar valued functional $\mathcal{F}$, with respect to its argument $x$

$\log \quad=\quad \exp ^{-1} ;$ logarithm map

$x_{j \mid k}=$ predicted variable $x_{k+j}$, predicted at time $k$; note that $x_{0 \mid k}$ is equal to $x_{k}$

$\langle\cdot, \cdot\rangle \quad=$ standard inner product on $\mathbb{R}^{3}$

$\langle\langle\cdot, \cdot\rangle\rangle=$ natural pairing between elements in $\mathfrak{s o}(3)$ and $\mathfrak{s o}(3)^{*}$

Superscripts

$\times \quad=\quad$ cross map from $\mathbb{R}^{3}$ to $\mathfrak{s o}$ (3); if $C$ is equal to $a^{\times}$then $C b$ is equal to $a \times b$ for all $b \in \mathbb{R}^{3}$, where $\times$ is the cross product

$-\times \quad=\quad$ inverse of the cross map
Received 4 December 2015; revision received 26 August 2016; accepted for publication 8 October 2016; published online 23 December 2016. Copyright $\odot 2016$ by the American Institute of Aeronautics and Astronautics, Inc. All rights reserved. All requests for copying and permission to reprint should be submitted to CCC at www.copyright.com; employ the ISSN 0731-5090 (print) or 1533-3884 (online) to initiate your request. See also AIAA Rights and Permissions www.aiaa.org/randp.

*Postdoctoral Fellow, Center for Space Research; daylee@umich.edu.

'Department of Aerospace Engineering; rohitgpt@ umich.edu.

łkalabic@umich.edu.

\$dicairano@merl.com.

†Professor, Department of Mathematics; abloch@umich.edu.

**Associate Professor, Department of Aerospace Engineering; jwcutler@ umich.edu.

${ }^{\dagger}$ Professor, Department of Aerospace Engineering; ilya@umich.edu. Member AIAA.

\section{Introduction}

$\mathbf{I}^{\mathrm{N}}$ N RECENT years, increasing demand for downsized and more agile spacecraft has led to a need for control techniques that can ensure safe and reliable spacecraft operation at the limits of performance. These techniques must take system constraints into account while maximizing performance. One such technique is model predictive control (MPC) [1] , which solves a constrained optimization problem and yields a stabilizing constraint-admissible control law. When MPC is based on linear models, the optimization problem becomes a constrained quadratic programming problem, which can be solved quickly using processing power that is available on board; for this reason, MPC based on linear models is often used when fast computations are desired. For examples of such an approach to spacecraft attitude control, see [2-6]. MPC based on nonlinear models used for prediction and optimization and/or nonlinear constraints is called nonlinear MPC (NMPC). If the constrained system is nonlinear, then the use of a nonlinear model in the NMPC scheme leads to more realistic predictions, and therefore to potentially better performance over linear MPC. Because the NMPC optimization problem cannot be reduced to a quadratic programming problem, other numerical methods are needed in order to quickly solve for the NMPC control. In this work, we present an NMPC algorithm for use in constrained spacecraft attitude control.

Constrained spacecraft attitude control refers to the control of spacecraft attitude in the presence of state and control constraints, which include constraints on the actuation mechanisms, the spacecraft orientation, its angular velocity, etc. For instance, reaction wheels used as attitude control actuators in many spacecraft can only generate limited torque and have maximum angular speed limits. The violation of these actuator constraints can cause instability or the loss of pointing accuracy. Despite the aforementioned issues, the reaction wheels are still popular as the main actuators for attitude control purposes in many small satellites because they are powered by electricity and not fuel and are easier to build and integrate in as compared to thrusters. So, in this paper, we develop a control algorithm that can be applied to a spacecraft with a reaction wheel assembly (RWA). Star trackers used for onboard attitude determination induce exclusion-zone constraints because star trackers cannot measure the spacecraft attitude when the sun or the Earth is within the camera angle and because long exposure to the sun can damage the camera sensor. Similarly, an accurate sun vector 
measurement from multiple photodiodes is required to measure the spacecraft attitude. However, in many small satellites, the photodiodes have limited install angles and positions that make it difficult to ensure the measurement of the sun vector all the time [7]. Small satellite attitude determination and control system engineers sometimes select locations and angles for the photodiodes that only guarantee a high probability of being exposed to the sun but still leave "dark spots" where the sun vector cannot be detected. Constrained control can be used to avoid entering in such dark spots where attitude determination becomes impossible. Furthermore, most small spacecraft have several design issues originating from limited resources such as photodiode installation, etc., which also motivates the use of constrained control.

Various approaches to handle actuator constraints were proposed in [8-11], whereas exclusion-zone constraints were treated in [12-17]. For example, attitude control problems with exclusion-zone constraints were addressed in the framework of open-loop attitude planning and commanding in $[\underline{15}, \underline{17}]$ and in exploiting feedback control in $[12,16]$.

As stated previously, in this paper, we use an NMPC approach to enforce the aforementioned constraints. The NMPC scheme was previously developed in [18] for spacecraft attitude, for which the dynamics evolved on a special orthogonal group in three dimensions $[\mathrm{SO}(3)]$ and for which the control inputs were external torques. The NMPC scheme from [18] was different from other NMPC approaches because the prediction model was obtained through the Lie group variational integrator (LGVI): LGVI is an integration scheme that, unlike standard integration schemes such as the RungeKutta, preserves conserved quantities of motion, and therefore leads to a more accurate prediction model. The dynamics obtained via the LGVI evolved on Lie groups, of which SO(3) was an example. The NMPC approach of [18] used the LGVI spacecraft attitude prediction model in order to enforce system constraints. Note that the work in [18] was generalized to systems for which the dynamics evolved on general manifolds [19]. In [20], the authors developed a numerical solver for the NMPC problem of [18]. The solver was based on [21] and used exterior penalty function for constraint handling. This paper is an extension of the approach in $[18,20]$ to the case of spacecraft with a RWA. The use of MPC for spacecraft attitude control was supported by the fact that, as shown in $[18,19]$, MPC can provide global asymptotic stability for systems for which the dynamics evolved on $\mathrm{SO}(3)$. This is in spite of the fact that, for the same systems, there do not exist globally asymptotically stabilizing continuous control laws.

The extension to spacecraft with a RWA is significant because this case is complicated by the need to consider reaction wheel speeds and the angular momentum exchange between the wheels and the spacecraft bus in formulating the dynamic model of the spacecraft, as well as in solving the NMPC problem numerically. Moreover, from a practical standpoint, the case of spacecraft with reaction wheels is important. Reaction wheels are used in many spacecraft and, unlike thrusters, reaction wheels can be used for precise pointing and attitude tracking while not consuming any fuel. We assume that reaction wheel desaturation is performed, if necessary, by external torques such as magnetometers or thrusters. The combined treatment of reaction wheels and external actuators in MPC design is also possible following the same approach, but the details, including necessary conditions for optimality and sensitivity calculations, are more involved and left to future publications. Other reaction wheel configurations, such as a tetrahedral configuration, can be treated as well by appropriately reflecting the reaction wheel configuration in the $\mathcal{S}$ matrix used in the subsequent developments. In fact, the relative ease by which different reaction wheel configurations can be accommodated is an appealing characteristic of our approach. The use of NMPC based on an LGVI model is pursued because, despite a potentially higher computational cost, it exploits a more realistic model of the spacecraft dynamics, which can provide an increased constrained domain of attraction. For example, when the linearized attitude dynamics are used for prediction in MPC, the achievement of rest-to-rest maneuvering is affected by the initial attitude condition of the spacecraft [22]. If the initial attitude is outside the domain of attraction, the maneuver is not achieved to the target (by definition, if the initial attitude is outside of the domain of attraction, the closedloop trajectories do not converge). By adopting the nonlinear LGVI model in MPC, we can achieve a larger, and possibly global [19], domain of the attraction. This topic is treated more in the Simulations and Discussion section (Sec. V). As in [20], the indirect single shooting method is applied to the nonlinear root finding problem resulting from the necessary conditions for optimality, which is warm started from the solution at the previous time instant. Our implementation also exploits sensitivity derivative expressions obtained from the necessary conditions for optimality, derived using techniques of calculus of variations on Lie groups. The constraints are handled using an exterior penalty function approach; such an implementation is advantageous to handle state-constraint infeasibility that may occur during real operation due to disturbances or model mismatch, and it does not introduce extra variables that an implementation with slack variables may entail. In this paper, we investigate separately the two cases when the control torque is external and when the control torque is provided by the RWA. Clearly, one can combine the two approaches to exploit both external and internal (RWA) torques. The price to pay is in an increased complexity of the optimal control problem which leads to more complex necessary conditions for optimality and sensitivity calculations. Thus, we do not consider both external and internal torques at the same times in this paper. Because we focus on attitude control for a small satellite, we place more emphasis on the RWA torques because, in small satellites, it is often impossible to install external torque generating devices, such as thrusters. Furthermore, external torques generated by magnetorquers are also much weaker than the torques from a RWA, and they are not efficient for precise and fast attitude control. This paper includes the concurrent handling of reaction wheel constraints and attitude constraints, as well as a specific application to a spacecraft with a RWA. As previously mentioned, these actuator constraints and the attitude constraint are becoming more critical in the recent missions, including several university satellites. The suggested algorithm can be applied to any configuration of the RWA and can be extended to other actuators based on the exchange of angular momentum. It is expected that it would be possible to execute the proposed algorithm in real time (after conversion to a $\mathrm{C}$ code) and using an embedded processor.

This paper is organized as follows. In Sec. II, discrete dynamics on the SO(3) are introduced for the spacecraft. In Sec. III, we define the NMPC problem. In Sec. IV, we present our computational solver. To demonstrate the effectiveness of the proposed algorithm, simulation results are presented in Sec. V. The conference paper of [23] included preliminary results. As compared to [23], this paper is longer and contains details and derivations not presented in [23], including the treatment of a practical spacecraft configuration with four reaction wheels. Note that the approach of our NMPC solver (based on geometric mechanics formalism) can also be extended to other problems of a similar kind, e.g., problems with different reaction wheel configurations or problems of combined transitional and rotational motion control [24]. We also present the comparison of the MPC problem formulations and numerical solution procedure in the case of the spacecraft with a RWA and spacecraft with external moments. Although small satellites mostly control attitude via internal torques of angular momentum exchanging devices, large satellites may rely on external control torques from thrusters when attitude pointing requirements are not very stringent. Thus, we include the case of spacecraft with external torques to demonstrate how the proposed approach is applicable to different satellites with relatively small modifications.

\section{Discrete Dynamics of a Spacecraft on SO(3)}

In this section, we present the discrete-time rigid-body equations of motion for a satellite with and without a reaction wheel assembly. In both cases, the dynamics are obtained by using the LGVI. The equations of motion for a spacecraft with external control moments, derived in [25], are given by 


$$
\begin{aligned}
h \Pi_{k}^{\times} & =F_{k} J_{d}-J_{d} F_{k}^{T} \\
R_{k+1} & =R_{k} F_{k} \\
\Pi_{k+1} & =F_{k}^{T} \Pi_{k}+h M_{k}+h u_{k}
\end{aligned}
$$

In the preceding equation, $h$ is the sampling time; the matrices $R_{k}$ and $F_{k}$ are rotation matrices and elements of $\mathrm{SO}(3)$, where $R_{k}$ is the spacecraft orientation and $F_{k}=R_{k}^{T} R_{k+1}$ is the difference in orientation between samples; $\Pi_{k} \in \mathbb{R}^{3}$ is the spacecraft total angular momentum; $M_{k} \in \mathbb{R}^{3}$ is the vector of external disturbances that results from gravity gradients, aerodynamic drag, solar pressure, etc.; $u_{k} \in \mathbb{R}^{3}$ is the control torque, which is used to stabilize the spacecraft attitude; and the positive definite matrix $J_{d}$ is the nonstandard moment of inertia matrix and is related to the standard inertia matrix $J$ by the equation,

$$
J_{d}=\frac{1}{2} \operatorname{tr}(J) I_{3}-J
$$

Note that $J$ is the inertia matrix of the whole spacecraft and includes the inertia of reaction wheels. Also, note that, in Eq. (1), the implicit equation [Eq. (1 $\underline{1 \mathrm{a}})$ ] is solved first to obtain $F_{k}$; then, $R_{k+1}$ and $\Pi_{k+1}$ are obtained using Eqs. (1b) and (1c).

Next, we consider a spacecraft with a RWA but without external control torques. A spacecraft with a RWA controls its attitude by adjusting the angular velocity of its reaction wheels in order to induce a counter rotation of the spacecraft. Accordingly, the LGVI equations of motion for a spacecraft with a RWA differ from Eq. (1) because the actuation mechanisms apply a torque about the reaction wheels instead of applying a torque directly to the spacecraft. Following the derivations in [26], we obtain the following equations of motion:

$$
\begin{aligned}
h \Pi_{k}^{\times} & =F_{k} A_{k}-A_{k}^{T} F_{k}^{T} \\
R_{k+1} & =R_{k} F_{k} \\
\Pi_{k+1} & =F_{k}^{T} \Pi_{k}+h M_{k}
\end{aligned}
$$

which are similar to those in Eq. (1), along with equations of motion relating to the reaction wheel assembly:

$$
\begin{aligned}
A_{k} & =J_{d}+\frac{1}{2} \sum_{i=1}^{n}\left(J_{i} \Delta \theta_{i, k} s_{i}\right)^{\times} \\
\pi_{i, k+1} & =\pi_{i, k}+h \tau_{i, k} \\
h \pi_{i, k} & =J_{i}\left(\Delta \theta_{i, k}-\frac{1}{2} \operatorname{tr}\left(F_{k} s_{i}^{\times}\right)\right)
\end{aligned}
$$

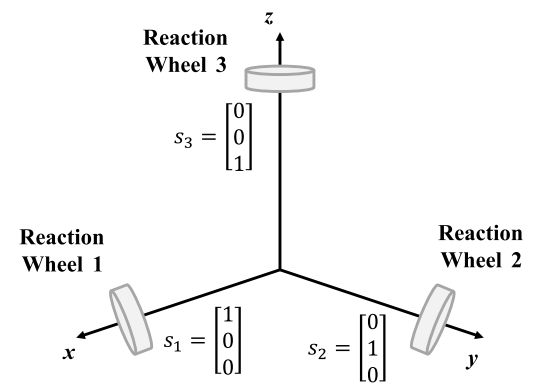

a) RWAwith three wheels
In the preceding equation, $n$ is the number of reaction wheels in the spacecraft with $i$ ranging from one to $n$. The main rotation direction vector of each reaction wheel is denoted by $s_{i}$; we assume that the rotor is a thin, flat disk and symmetric with respect to the rotation axis. The scalar $\pi_{i, k}$ is the angular momentum of the $i$ th wheel about the $s_{i}$ axis, where $s_{i}$ is a unit vector with origin at the spacecraft center of mass; $\Delta \theta_{i, k}$ is the difference in rotation wheel position between samples; $\tau_{i, k}$ is the control torque applied to the $i$ th wheel; and the parameter $J_{i}>0$ is the moment of inertia of the $i$ th wheel about its spin axis [26]. For illustration, in Fig. 1, we provide two examples of RWAs with three and four reaction wheels [27].

\section{Nonlinear Model Predictive Control Problem Formulation for a Spacecraft on SO(3)}

In this section, we present an NPMC scheme for constrained control of the spacecraft dynamics, subject to the equations of motion in Eq. (1) or Eqs. (3) and (4), which were introduced in the previous section. The NMPC scheme is based on the NMPC framework introduced in $[18,19]$ and uses the computationally fast solver, which is similar to the one presented in [20] for determining the control input.

For a spacecraft with external control torque, for which the dynamics are given in Eq. (1), the controller determines the control input by solving the following optimization problem:

$$
\min _{\left\{u_{j \mid k}\right\}_{j=0}^{N-1}} \mathcal{J}_{d}^{1}=K_{d}^{1}\left(R_{N \mid k}, \Pi_{N \mid k}\right)+\sum_{k=0}^{N-1} C_{d}^{1}\left(R_{j \mid k}, \Pi_{j \mid k}, u_{j \mid k}\right)
$$

subject to $h \Pi_{j \mid k}^{\times}=F_{j \mid k} J_{d}-J_{d} F_{j \mid k}^{T}$

$$
\begin{aligned}
& R_{j+1 \mid k}=R_{j \mid k} F_{j \mid k} \\
& \Pi_{j+1 \mid k}=F_{j \mid k}^{T} \Pi_{j \mid k}+h M_{j \mid k}+h u_{j \mid k} \\
& H_{\ell}^{1}\left(R_{j \mid k}, \Pi_{j \mid k}, u_{j \mid k}\right) \leq 0, \quad \ell=1, \ldots, m
\end{aligned}
$$

In the preceding equation, the dynamics in Eq. (2) were included as equality constraints. Other inequality constraints are introduced in Eq. (5e). These inequality constraints can include pointing, exclusion-zone constraints, constraints on the available torque, and so on. Note that $m$ is the number of inequality constraints considered. In the NMPC law, the control input $u_{k}$ to Eq. (1) is set to the first element of the control sequence

$$
\left\{u_{j \mid k}^{*}\right\}_{j=0}^{N-1}
$$

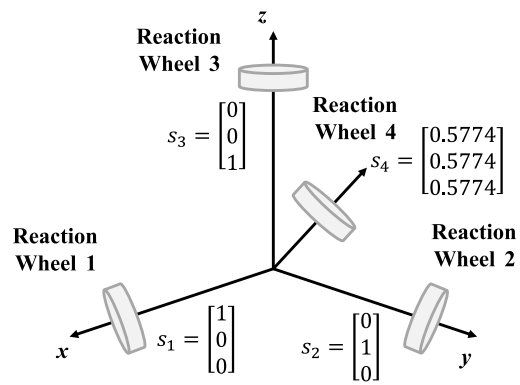

b) RWAwith four wheels

Fig. 1 Two different RWA configurations and their spin axes. 
solving Eq. (ㅁ), i.e.,

$$
u_{k}=u_{0 \mid k}^{*}
$$

The functions $K_{d}^{1}$ and $C_{d}^{1}$ satisfy the assumptions given in [19]. We note that, in [19], terminal conditions were imposed to guarantee recursive feasibility and stability. These constraints were stringent and greatly reduced the domain of attraction in general, thereby degrading the closed-loop response; hence, constraints were treated as soft. We rely on simulation-based verification of stability properties.

For the dynamics [Eqs. (3) and (4)] relating to a spacecraft with a RWA, the NMPC optimization problem is given by

$$
\min _{\left\{\tau_{j \mid k}\right\}_{j=0}^{N-1}} \mathcal{J}_{d}^{2}=K_{d}^{2}\left(R_{N \mid k}, \Pi_{N \mid k}, \pi_{j \mid k}\right)+\sum_{k=0}^{N-1} C_{d}^{2}\left(R_{j \mid k}, \Pi_{j \mid k}, \pi_{j \mid k}, \tau_{j \mid k}\right)
$$

subject to $h \Pi_{j \mid k}^{\times}=F_{j \mid k} A_{j \mid k}-A_{j \mid k}^{T} F_{j \mid k}^{T}$

$$
R_{j+1 \mid k}=R_{j \mid k} F_{j \mid k}
$$

$$
\Pi_{j+1 \mid k}=F_{j \mid k}^{T} \Pi_{j \mid k}+h M_{j \mid k}
$$

$$
\pi_{j+1 \mid k}=\pi_{j \mid k}+h \tau_{j \mid k}
$$

$$
H_{\ell}^{2}\left(R_{j \mid k}, \Pi_{j \mid k}, \pi_{j \mid k}, \tau_{j \mid k}\right) \leq 0, \quad \ell=1, \ldots, m
$$

The treatment of this optimization problem is analogous to that of Eq. (5), with the functions $K_{d}^{2}, C_{d}^{2}$, and $H_{\ell}^{2}$ satisfying properties analogous to those of $K_{d}^{1}, C_{d}^{1}$, and $H_{\ell}^{1}$, respectively.

In practice, constraints may become infeasible due to disturbance or modeling error. To ensure controller operation in the event of constraint violation, we relax Eqs. (5e) and (7f) and treat them as soft. Then, the numerical solution is based on the necessary conditions for optimality for augmented cost functionals of $\mathcal{J}_{d, a}^{1}$ and $\mathcal{J}_{d, a}^{2}$. The augmented cost functional corresponding to the cost functional $\mathcal{J}_{d, a}^{1}$ is given by

$$
\begin{aligned}
& \mathcal{J}_{d, a}^{1}=K_{d}^{1}\left(R_{N}, \Pi_{N}\right)+\sum_{k=0}^{N-1} C_{d}^{1}\left(R_{k}, \Pi_{k}, u_{k}\right) \\
& +\sum_{k=0}^{N-1}\left\langle\lambda_{k}^{1},\left(\log \left(R_{k}^{-1} R_{k+1}\right)-\log \left(F_{k}\right)\right)^{-\times}\right\rangle \\
& +\sum_{k=0}^{N-1}\left\langle\lambda_{k}^{2},\left(\Pi_{k+1}-F_{k}^{T} \Pi_{k}-h M_{k}-h B u_{k}\right)\right\rangle \\
& +\sum_{k=0}^{N-1} \sum_{\ell=1}^{m} \mu_{\ell} \phi_{\ell}\left(H_{\ell}^{1}\left(R_{k}, \Pi_{k}, u_{k}\right)\right)
\end{aligned}
$$

where we have introduced the Lagrange multipliers $\lambda_{k}^{1}, \lambda_{k}^{2} \in \mathbb{R}^{3}$; the exterior penalty functions $\phi_{\ell}, \ell=1, \ldots, m$; and the scalar weighting factors $\mu_{\ell}>0, \ell=1, \ldots, m$. The augmented cost functional corresponding to $\mathcal{J}_{d, a}^{2}$ is given by

$$
\begin{aligned}
& \mathcal{J}_{d, a}^{2}=K_{d}^{2}\left(R_{N}, \Pi_{N}, \pi_{N}\right)+\sum_{k=0}^{N-1} C_{d}^{2}\left(R_{k}, \Pi_{k}, \pi_{k}, \tau_{k}\right) \\
& +\sum_{k=0}^{N-1}\left\langle\lambda_{k}^{1},\left(\log \left(R_{k}^{-1} R_{k+1}\right)-\log \left(F_{k}\right)\right)^{-\times}\right\rangle \\
& +\sum_{k=0}^{N-1}\left\langle\lambda_{k}^{2},\left(\Pi_{k+1}-F_{k}^{T} \Pi_{k}-h M_{k}\right)\right\rangle \\
& +\sum_{k=0}^{N-1}\left\langle\lambda_{k}^{3},\left(\pi_{k+1}-\pi_{k}-h \tau_{k+1}\right)\right\rangle \\
& +\sum_{k=0}^{N-1} \sum_{\ell=0}^{m} \mu_{\ell} \phi_{\ell}\left(H_{\ell}^{2}\left(R_{k}, \Pi_{k}, \pi_{k}, \tau_{k}\right)\right)
\end{aligned}
$$

where $\lambda_{k}^{3} \in \mathbb{R}^{3}$ is a Lagrange multiplier.

\section{Description of the Numerical Solver}

The numerical solver is based on solving the necessary conditions for optimality. In deriving the necessary conditions, we follow the approach in [20] and use calculus of variations on Lie groups in the discrete-time setting. The necessary conditions for optimality are given by

$$
\begin{aligned}
& h \Pi_{k}^{\times}=F_{k} A_{k}-A_{k}^{T} F_{k}^{T} \\
& R_{k+1}=R_{k} F_{k} \\
& \Pi_{k+1}=F_{k}^{T} \Pi_{k}+h M_{k} \\
& \pi_{k+1}=\pi_{k}+h \tau_{k} \\
& {\left[\begin{array}{l}
\lambda_{k}^{1} \\
\lambda_{k}^{2} \\
\lambda_{k}^{3}
\end{array}\right]=\left[\begin{array}{lll}
\mathcal{A}_{k+1}^{T} & \mathcal{C}_{k+1}^{T} & 0_{3 \times n} \\
\mathcal{B}_{k+1}^{T} & \mathcal{D}_{k+1}^{T} & 0_{3 \times n} \\
\mathcal{E}_{k+1}^{T} & \mathcal{G}_{k+1}^{T} & I_{n \times n}
\end{array}\right]\left[\begin{array}{c}
\lambda_{k+1}^{1} \\
\lambda_{k+1}^{2} \\
\lambda_{k+1}^{3}
\end{array}\right]-\left[\begin{array}{c}
\mathcal{H}_{k+1} \\
\mathcal{L}_{k+1} \\
\mathcal{P}_{k+1}
\end{array}\right] } \\
& h \lambda_{k}^{3}= D_{\tau_{k}} C_{d}+\sum_{\ell=1}^{m} \mu_{\ell} D_{\tau_{k}}\left(\phi_{\ell} \circ H_{\ell}\right)
\end{aligned}
$$

where

$$
\begin{aligned}
\mathcal{A}_{k} & =F_{k}^{T} \\
\mathcal{B}_{k} & =h\left(\left(\operatorname{tr}\left(F_{k} A_{k}\right) I_{3 \times 3}-F_{k} A_{k}\right) F_{k}-\frac{1}{4}\left(\operatorname{tr}\left(F_{k}\right) I_{3 \times 3}-F_{k}^{T}\right)\right. \\
& \left.\mathcal{S}\left(J_{w}\right) \mathcal{S}^{T}\left(\operatorname{tr}\left(F_{k}\right) I_{3 \times 3}-F_{k}^{T}\right)\right)^{-1} \\
\mathcal{C}_{k} & =h \mathcal{M}_{k} \\
\mathcal{D}_{k} & =F_{k}^{T}+\left(F_{k}^{T} \Pi_{k}\right)^{\times} \mathcal{B}_{k} \\
\mathcal{E}_{k} & =-\mathcal{B}_{k}\left(\frac{1}{2}\left(\operatorname{tr}\left(F_{k}\right) I_{3 \times 3}-F_{k}^{T}\right) \mathcal{S}\right) \\
\mathcal{G}_{k} & =\left(F_{k}^{T} \Pi_{k}\right)^{\times} \mathcal{E}_{k} \\
\mathcal{H}_{k} & =\left(\left(R_{k}^{T}\left(D_{R_{k}} C_{d}^{2}\right)\right)_{A}\right)^{-\times}+\sum_{\ell=1}^{m} \mu_{\ell}\left(\left(R_{k}^{T}\left(D_{R_{k}}\left(\phi_{\ell} \circ H_{\ell}\right)\right)\right)_{A}\right)^{-\times} \\
\mathcal{L}_{k} & =D_{\Pi_{k}} C_{d}^{2}+\sum_{\ell=1}^{m} \mu_{\ell} D_{\Pi_{k}}\left(\phi_{\ell} \circ H_{\ell}\right) \\
\mathcal{P}_{k} & =D_{\pi_{k}} C_{d}^{2}+\sum_{\ell=1}^{m} \mu_{\ell} D_{\Pi_{k}}\left(\phi_{\ell} \circ H_{\ell}\right)
\end{aligned}
$$


In the preceding equations, $\mathcal{S} \in \mathbb{R}^{3 \times n}$ (which is the reaction wheel configuration matrix), and $J_{w} \in \mathbb{R}^{n \times n}$ (which is the rotational inertia matrix of the RWA) are defined by

$$
\begin{gathered}
\mathcal{S}=\left[\begin{array}{lll}
s_{1} & \cdots & s_{n}
\end{array}\right] \\
J_{w}=\left[\begin{array}{ccc}
J_{1} & 0 & 0 \\
0 & \ddots & 0 \\
0 & 0 & J_{n}
\end{array}\right]
\end{gathered}
$$

Note that the composition of the $\mathcal{S}$ matrix is arbitrary, and the reaction wheels do not need to be aligned to the principal axis of the spacecraft. The details of the derivation are available in the Appendix.

To solve the two-point boundary value problem [Eq. (10)], the indirect single shooting method is used, where the goal is to determine the initial values of the Lagrange multipliers; we follow a similar approach to that found in $[\underline{20}, \underline{21}]$. The method calculates the sensitivity derivative of the terminal condition error with respect to the initial Lagrange multiplier, which is used as the Jacobian matrix. The sensitivity derivatives for Eqs. (10b-10d) are given by

$$
\left[\begin{array}{c}
\zeta_{k+1} \\
\delta \Pi_{k+1} \\
\delta \pi_{k+1}
\end{array}\right]=\left[\begin{array}{ccc}
\mathcal{A}_{k} & \mathcal{B}_{k} & \mathcal{E}_{k} \\
\mathcal{C}_{k} & \mathcal{D}_{k} & \mathcal{G}_{k} \\
0_{n \times 3} & 0_{n \times 3} & I_{n \times n}
\end{array}\right]\left[\begin{array}{c}
\zeta_{k} \\
\delta \Pi_{k} \\
\delta \pi_{k}
\end{array}\right]+\left[\begin{array}{c}
0_{3 \times n} \\
0_{3 \times n} \\
h I_{n \times n}
\end{array}\right] \delta \tau_{k}
$$

whereas the sensitivity derivatives for Eq. (10e) are given by

$$
\left[\begin{array}{l}
\delta \lambda_{k+1}^{1} \\
\delta \lambda_{k+1}^{2} \\
\delta \lambda_{k+1}^{3}
\end{array}\right]=S_{k}\left[\begin{array}{c}
\zeta_{k+1} \\
\delta \Pi_{k+1} \\
\delta \pi_{k+1} \\
\delta \tau_{k} \\
\delta \lambda_{k}^{1} \\
\delta \lambda_{k}^{2} \\
\delta \lambda_{k}^{3}
\end{array}\right]
$$

where $S_{k}$ is a $(6+n) \times(12+3 n)$ matrix. Expressing $\delta \tau_{k}$ in terms of $\tau_{k}$ and $\delta \lambda_{k}^{3}$, from Eqs. (13) and (14), we obtain

$$
\left[\begin{array}{c}
\zeta_{k+1} \\
\delta \Pi_{k+1} \\
\delta \pi_{k+1} \\
\delta \lambda_{k+1}^{1} \\
\delta \lambda_{k+1}^{2} \\
\delta \lambda_{k+1}^{3}
\end{array}\right]=T_{k}\left[\begin{array}{c}
\zeta_{k} \\
\delta \Pi_{k} \\
\delta \pi_{k} \\
\delta \lambda_{k}^{1} \\
\delta \lambda_{k}^{2} \\
\delta \lambda_{k}^{3}
\end{array}\right]
$$

where $T_{k}$ is an $(12+2 n) \times(12+2 n)$ matrix. Therefore, from Eq. (15), we obtain:

$$
\left[\begin{array}{c}
\zeta_{N} \\
\delta \Pi_{N} \\
\delta \pi_{N} \\
\delta \lambda_{N}^{1} \\
\delta \lambda_{N}^{2} \\
\delta \lambda_{N}^{3}
\end{array}\right]=\left(\prod_{k=0}^{N-1} T_{k}\right)\left[\begin{array}{c}
\zeta_{0} \\
\delta \Pi_{0} \\
\delta \pi_{0} \\
\delta \lambda_{0}^{1} \\
\delta \lambda_{0}^{2} \\
\delta \lambda_{0}^{3}
\end{array}\right]
$$

To determine the initial values of the Lagrange multipliers, we employ the indirect single shooting method, the iterations of which have the following form:

$$
\lambda_{0}^{(p+1)}=\lambda_{0}^{(p)}-\gamma\left[\frac{\delta E^{(p)}}{\delta \lambda_{0}^{(p)}}\right]^{-1} E^{(p)}
$$

where the superscripts represent the iteration number, the step size $\gamma$ is a scalar satisfying $0<\gamma \leq 1$, and $E^{(p)}$ and $\delta E^{(p)}$ are given as follows:

$$
\begin{aligned}
E^{(p)}= & {\left[\begin{array}{c}
\lambda_{N-1}^{1(p)}+\left(\left(R_{N}^{T}\left(D_{R_{N}} K_{d}^{1,2}\right)\right)_{A}\right)^{-\times(p)} \\
\lambda_{N-1}^{2(p)}+D_{\Pi_{N}} K_{d}^{1,2(p)} \\
\lambda_{N-1}^{3(p)}+D_{\pi_{N}} K_{d}^{1,2(p)}
\end{array}\right], } \\
\delta E^{(p)}= & {\left[\begin{array}{c}
\delta \lambda_{N-1}^{1(p)}+\left(\left(\delta R_{N}^{T}\left(D_{R_{N}} K_{d}^{1,2}\right)+R_{N}^{T}\left(\delta D_{R_{N}} K_{d}^{1,2}\right)\right)_{A}\right)^{-\times(p)} \\
\delta \lambda_{N-1}^{2(p)}+\delta D_{\Pi_{N}} K_{d}^{1,2(p)} \\
\delta \lambda_{N-1}^{3(p)}+\delta D_{\pi_{N}} K_{d}^{1,2(p)}
\end{array}\right] }
\end{aligned}
$$

The numerical solver is summarized in Fig. 2. During each sampling period, the solver determines the Lagrange multipliers so that the terminal boundary conditions are satisfied. During the initialization step (step (1) in Fig. 2), the initial value of Eq. (17) is set to the solution from the previous sampling time. The numerical solver then updates the values of the Lagrange multipliers using the necessary conditions for optimality in step (2). The shooting method proceeds through steps (4), (5), and (6) until the termination criterion is satisfied in step (3) for $\epsilon=10^{-5}$. Note that the parameter $\gamma$ is initially set at one; if, after 50 iterations (i.e., $p=50$ ), Eq. (17) has not converged, then the algorithm sets $\gamma=0.1$. The initial guess of the Lagrange multiplier at the first step is set to zero and, after that, it is warm started using the results of the previous step.

Remark 1: Our solution to the NMPC problem is based on the exterior penalty function approach and the indirect single shooting method, which is straightforward in terms of computational implementation when the equations of motion are formulated based on the LGVI. Other techniques, such as multiple shooting, interior point, or sequential quadratic programming, may also be of interest for future applications.

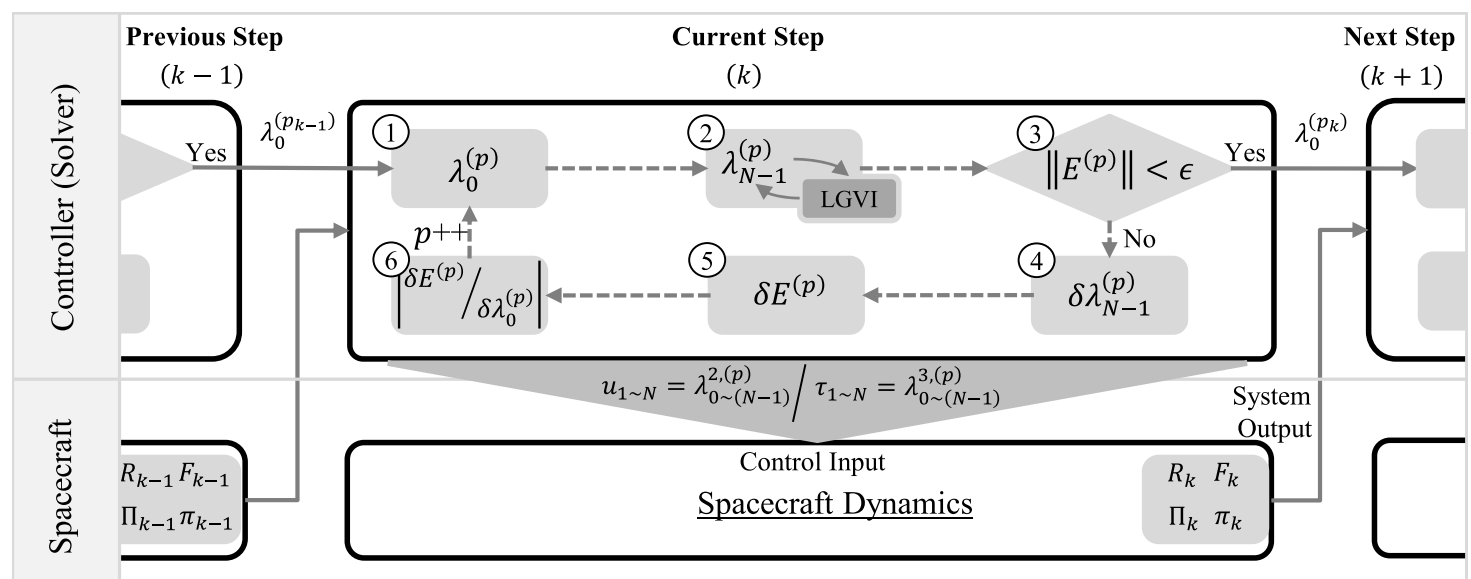

Fig. 2 Logic diagram for the numerical solver. 


\section{Simulations and Discussion}

In this section, we report simulation results for three cases. The first and the second cases correspond to spacecraft with the two different reaction wheel configurations that were presented in Fig. 1, and the last case corresponds to a spacecraft with external control moments, with equations of motion given in Eq. (1).

\section{A. Cost Functions and Inequality Constraints}

Cost functions $K_{d}^{1,2}$ and $C_{d}^{1,2}$ and constraint functions $H_{\ell}^{1,2}$ corresponding to the three simulations are presented in Table 1 . Matrices $P_{1}, P_{2}, Q_{1}, Q_{2}$, and $Q_{3}$ are symmetric positive-definite matrices. The constraints prescribed by $H_{1}^{1,2}$ correspond to the maximum available control torque $u_{k}$ or $\tau_{k}$, where $u_{k}=\left[\begin{array}{lll}u_{1, k} & u_{2, k} & u_{3, k}\end{array}\right]^{T}$ and $\tau_{k}=\left[\begin{array}{lll}\tau_{1, k} & \cdots & \tau_{n, k}\end{array}\right]^{T}$. The constraints prescribed by $H_{2}^{1,2}$ correspond to an inclusion-/exclusionzone constraint, where the cosine of the angle between the unit vectors $R_{k} v$ and $w$ must be greater than $\beta$. The final constraint that we consider is $H_{3}^{2}$, which is the maximum limit on the allowable rotation wheel angular momenta $\pi_{k}$, where $\pi_{k}=\left[\begin{array}{lll}\pi_{1, k} & \cdots & \pi_{n, k}\end{array}\right]^{T}$; the limit is denoted by $\chi$.

The differentiable penalty functions $\phi_{\ell}$ are defined through

$$
\phi_{\ell} \circ H_{\ell}^{1,2}=h \max \left(0, H_{\ell}^{1,2}\right)^{2}, \quad \ell=1,2,3
$$

\section{B. Simulation Results}

We consider a spacecraft with a moment of inertia matrix $J=\operatorname{diag}(1,1,0.8) \mathrm{kg} \cdot \mathrm{m}^{2}$. We set the integration time step to $h=0.4 \mathrm{~s}$, and we set the prediction horizon for the MPC algorithm to $N=5$. The weighting matrices are chosen as

$$
P_{1}=P_{2}=Q_{1}=Q_{2}=0.01 I_{3 \times 3}
$$

In some of the subsequent figures, the attitude maneuver is plotted on the two-sphere $\mathrm{S}^{2}$, where the vectors corresponding to the first, second, and third columns of $R_{0}$ are plotted in dashed lines, respectively. Similarly, the paths that are traced by the ends of the vectors corresponding to the first, second and third columns of $R_{k}$ are plotted in solid lines.

In the following, simulation results are reported for the cases of spacecraft with three and four reaction wheels.

\section{Spacecraft with RWA of Three Wheels}

We begin by considering a spacecraft with a RWA corresponding to the three-wheel configuration:

$$
\mathcal{S}=\left[\begin{array}{lll}
s_{1} & s_{2} & s_{3}
\end{array}\right]=\left[\begin{array}{lll}
1 & 0 & 0 \\
0 & 1 & 0 \\
0 & 0 & 1
\end{array}\right]
$$

Simulations of spacecraft attitude maneuvering are performed with and without constraints on the reaction wheel torque $H_{1}^{2}$, the exclusion/inclusion zone $H_{2}^{2}$, and the reaction wheel maximum angular momentum $H_{3}^{2}$. The constraint parameters are given by $\alpha=0.01, \quad \beta=\cos (170 \mathrm{deg}), \quad v=\left[\begin{array}{lll}1 & 0 & 0\end{array}\right]^{T}, \quad w=$ $-\left[\begin{array}{lll}0.8851 & 0.3888 & -0.2558\end{array}\right]^{T}$, and $\chi=0.05$. The penalty weights are chosen based on the process suggested in [28]. We increase the weight about one order of magnitude larger than the largest cost weight in the problem so that they are large enough to ensure that the constraints are enforced reasonably well. Their values are given by $\mu_{1}=10^{7}, \mu_{2}=10^{3}$, and $\mu_{3}=10^{4}$, which are determined by running multiple simulations. The initial conditions are chosen judiciously in order to ensure that the constraints become active during the simulation. The initial conditions for the attitude, the angular momentum of the spacecraft body, and the angular momentum of each reaction wheel are given by

$$
\begin{aligned}
& R_{0}=\exp \left(\zeta^{\times}\right), \quad \Pi_{0}=\left[\begin{array}{lll}
0 & 0 & 0
\end{array}\right]^{T}, \\
& \pi_{0}=\left[\begin{array}{lll}
0.008 & 0 & -0.015
\end{array}\right]^{T}
\end{aligned}
$$

where $\zeta=\left[\begin{array}{lll}0.5 & 0.5 & 0.5\end{array}\right]^{T}$. The inertia values of the reaction wheels are $J_{1}=J_{2}=J_{3}=0.01 \mathrm{~kg} \cdot \mathrm{m}^{2}$ and $Q_{3}=I_{3 \times 3}$. Both the constraint-free and constrained simulations are presented in Fig. $\underline{3}$. Referring to the subplots of Fig. 3 , we can see that the constraint-free simulation is stabilizing but violates the exclusion zone constraint in marked by (1), the torque constraints in marked by (3) and (4), and the angular momentum constraints in marked by (5) and (6). In the constrained simulation, all of these constraints are tightly enforced.

\section{Spacecraft with RWA of Four Wheels}

In the next case, we consider the four-reaction-wheel configuration corresponding to

$$
\mathcal{S}=\left[\begin{array}{llll}
s_{1} & s_{2} & s_{3} & s_{4}
\end{array}\right]=\left[\begin{array}{llll}
1 & 0 & 0 & 0.5774 \\
0 & 1 & 0 & 0.5774 \\
0 & 0 & 1 & 0.5774
\end{array}\right]
$$

The constraint parameters are changed to $\alpha=0.02, \beta=$ $\cos (170 \mathrm{deg}), \quad v=\left[\begin{array}{lll}1 & 0 & 0\end{array}\right]^{T}, \quad w=-\left[\begin{array}{lll}0.9624 & -0.2017 & 0.1818\end{array}\right]^{T}$, and $\chi=0.04$. The initial conditions are the same as in the previous case, except that the angular momentum of each reaction wheel is modified in order to ensure the activation of the angular momentum constraint. The reason a larger value of $\alpha$ has to be considered is that, with the addition of a fourth wheel and a change in the angular momentum initial conditions, the torque constraint from the previous simulation is found to be too stringent because the solver is unable to simultaneously satisfy all constraints. The other constraints are changed in order to modify the simulation scenario. The new initial conditions are given as follows:

$$
\pi_{0}=\left[\begin{array}{llll}
0.010 & 0 & 0.005 & -0.020
\end{array}\right]^{T}
$$

The inertia values of the reaction wheels are given by $J_{1}=J_{2}=$ $J_{3}=J_{4}=0.01 \mathrm{~kg} \cdot \mathrm{m}^{2}$ and $Q_{3}=I_{4 \times 4}$. The constraint-free and constrained simulation results are presented in Fig. 4 .

Referring to the subplots of Fig. 4, we can see that, although the exclusion zone constraint is violated in a situation marked by (1), it is enforced by the NMPC control law as marked by (2). Furthermore, we can see that the torque constraints are activated by control jumps marked by (3) and (4). These control torque jumps occur in order to prevent the violation of the zone constraint; and we note that, if the jump is deemed to be too large, its magnitude can be reduced by increasing the length of the prediction horizon $N$. In the next example, we consider the use of a longer prediction horizon.

Table 1 Summary of cost functions and inequality constraints

\begin{tabular}{ll}
\hline \hline Spacecraft with external control moments & Spacecraft with RWA \\
\hline$K_{d}^{1}=\frac{1}{2}\left\|P_{1}^{1 / 2}\left(R_{N}-I_{3 \times 3}\right)\right\|_{F}^{2}+\frac{1}{2} \Pi_{N}^{T} P_{2} \Pi_{N}$, & $K_{d}^{2}=\frac{1}{2}\left\|P_{1}^{1 / 2}\left(R_{N}-I_{3 \times 3}\right)\right\|_{F}^{2}+\frac{1}{2}\left(\Pi_{N}-\mathcal{S} \pi_{N}\right)^{T} P_{2}\left(\Pi_{N}-\mathcal{S} \pi_{N}\right)$, \\
$C_{d}^{1}=\frac{h}{2}\left\|Q_{1}^{1 / 2}\left(R_{k}-I_{3 \times 3}\right)\right\|_{F}^{2}+\frac{h}{2} \Pi_{N}^{T} Q_{2} \Pi_{N}+\frac{h}{2} u_{k}^{T} Q_{3} u_{k}$, & $C_{d}^{2}=\frac{h}{2}\left\|Q_{1}^{1 / 2}\left(R_{k}-I_{3 \times 3}\right)\right\|_{F}^{2}+\frac{h}{2}\left(\Pi_{k}-\mathcal{S} \pi_{k}\right)^{T} Q_{2}\left(\Pi_{k}-\mathcal{S} \pi_{k}\right)+\frac{h}{2} \tau_{k}^{T} Q_{3} \tau_{k}$, \\
$H_{1}^{1}=\left\|u_{k}\right\|_{2}-\alpha$, & $H_{1}^{2}=\left\|\tau_{k}\right\|_{2}-\alpha$, \\
$H_{2}^{1}=\beta-v^{T} R_{k}^{T} w$ & $H_{2}^{2}=\beta-v^{T} R_{k}^{T} w$, \\
& $H_{3}^{2}=\left\|\pi_{k}\right\|_{2}-\chi$ \\
\hline \hline
\end{tabular}



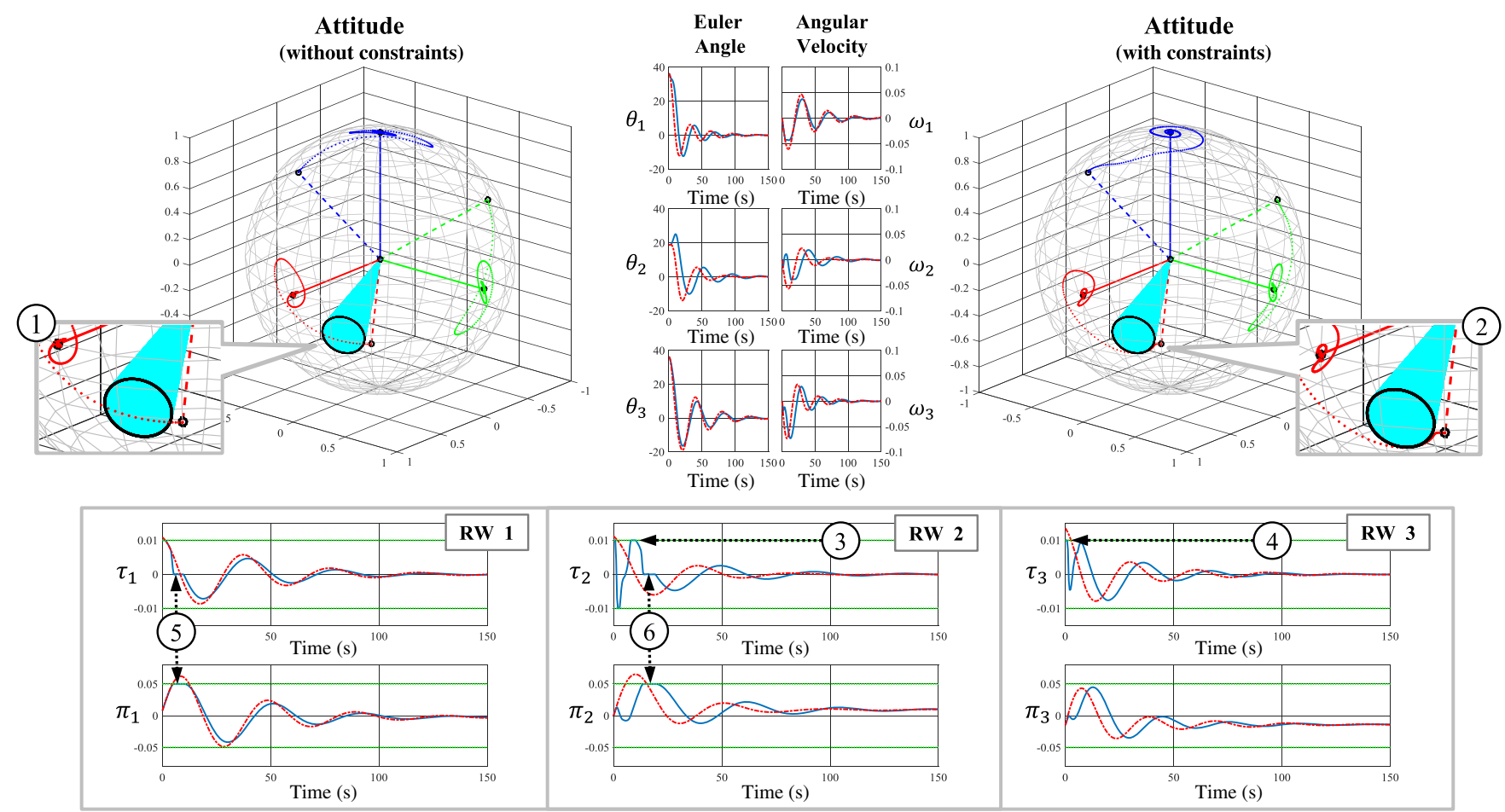

-With constraints -----Without constraints

Fig. 3 Model predictive control of three-RWA spacecraft with and without constraints.
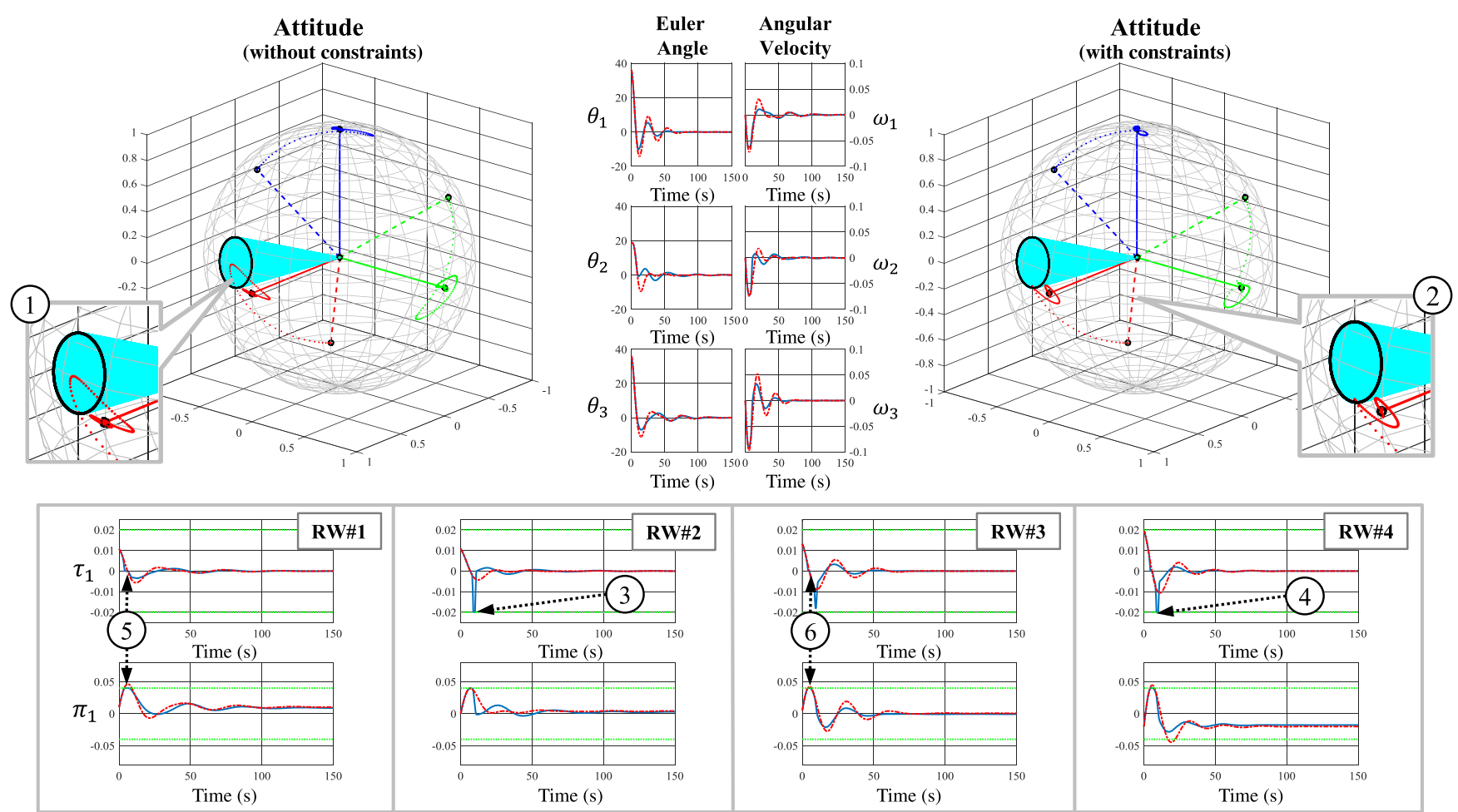

- With constraints -----Without constraints

Fig. 4 Model predictive control of four-RWA spacecraft with and without constraints.

\section{Spacecraft with External Control Moments}

A simulation of the spacecraft with external moments is performed with and without constraints only on the external moments and the exclusion/inclusion zone. As in the previous cases, we set the same integration time step at $h=0.4 \mathrm{~s}$, but the prediction horizon for the NMPC algorithm is now increased to $N=10$, allowing us to investigate the effect of a longer prediction horizon. The torque constraint constrain parameter is chosen to be $\alpha=0.05$, and the penalty weights are chosen to be $\mu_{1}=10^{4}$ and $\mu_{2}=5$. In the longer prediction horizon case, even with a weight lower than in the shorter horizon case, the controller can find a solution that satisfies the constraints. The initial conditions of the attitude, the body angular velocity, and the zone constraint are the same as those for the first simulation, corresponding to the three-wheel case of the spacecraft with a RWA. 

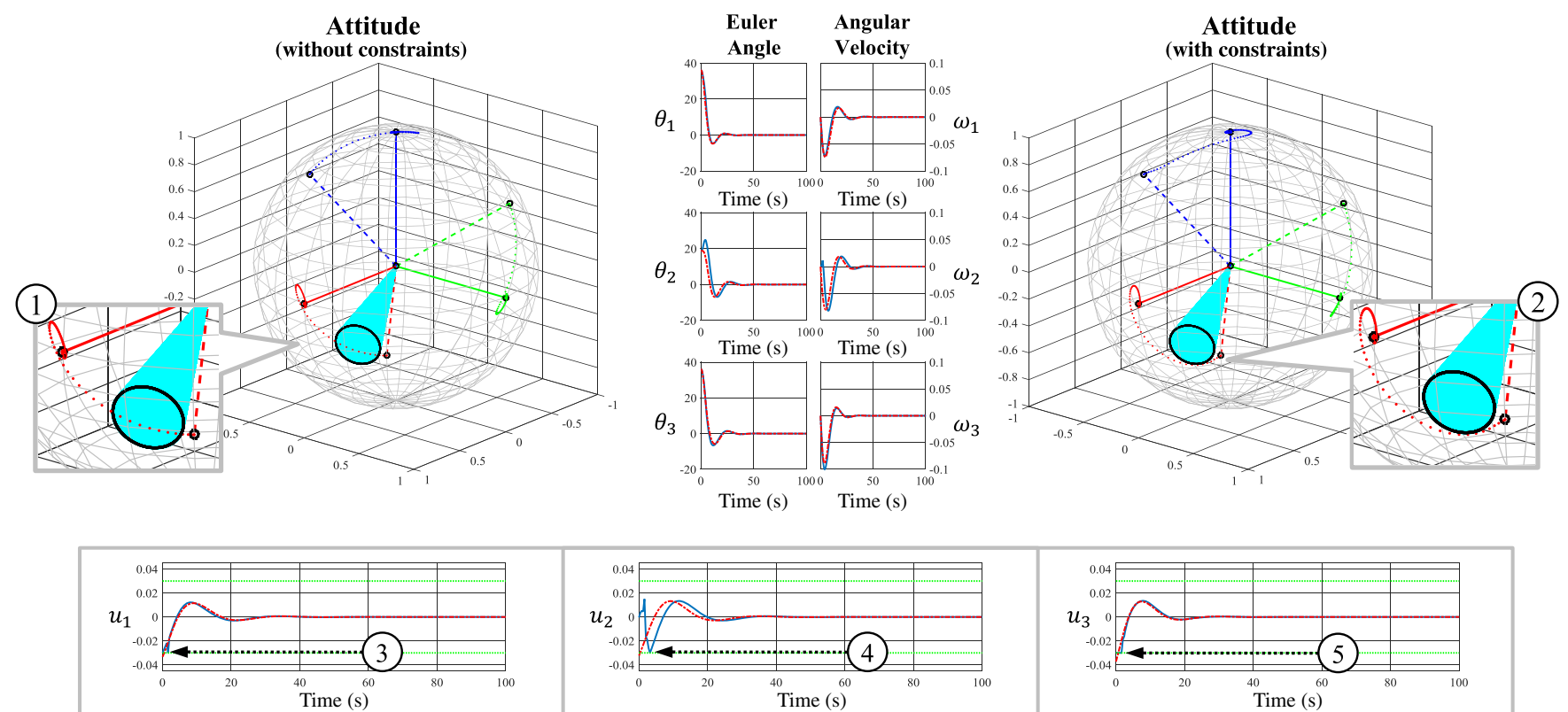

- With constraints ----- Without constraints

Fig. 5 Model predictive control of spacecraft moment with and without constraints.

The simulations results are presented in Fig. 5. Subplots marked by (1) and (2) in Fig. 5 show that the zone constraint can be satisfied by using the NMPC law, whereas subplots marked by (3), (4), and (5) show the satisfaction of the external moment constraint.

With the longer prediction horizon, the stabilization time and the overshoot in the attitude responses are shorter than in the previous simulations. This observation corresponds to simulations performed on the three- and four-wheel RWA configurations with longer prediction horizons. In general, a longer prediction horizon $N$ corresponds to a shorter stabilization time and smaller overshoot, but the NMPC computation takes longer to converge, especially in the complex case where there are many constraints. Two examples of the spacecraft with a RWA have a shorter prediction horizon (five steps) than the example of the spacecraft with external moments (ten steps). This is because we want to show that the suggested algorithm is capable of handling three different constraint categories simultaneously. When the spacecraft with a RWA has a zone constraint only, the longer prediction horizon works well and yields results similar to those of the example of the spacecraft with external moments. The longer prediction horizon reduces the overshoot and stabilization time but, if there exist different constraint categories in the problem, a shorter horizon can effectively prevent the simultaneous violation of multiple constraints.

\section{Domain of Attraction}

We now investigate the closed-loop domain of attraction of our NMPC algorithm by simulating 13,357 rest-to-rest orientation maneuvers for the spacecraft with a RWA. To show that the domain of attraction is global, or close to global, the simulations are subject only to maximum wheel torque constraints, with state constraints turned off, i.e., $H_{2}^{1,2}=H_{3}^{2} \equiv 0$. In the following, we list all combinations of 3-2-1 Euler angle parametrizations corresponding to the at-rest initial conditions that the algorithm is able to stabilize to the desired equilibrium $R_{k}=I_{3 \times 3}$ (all other state initial conditions are set to zero):

1) For roll, $-90,-80,-70, \cdots, 80,90 \operatorname{deg}(19$ cases $)$.
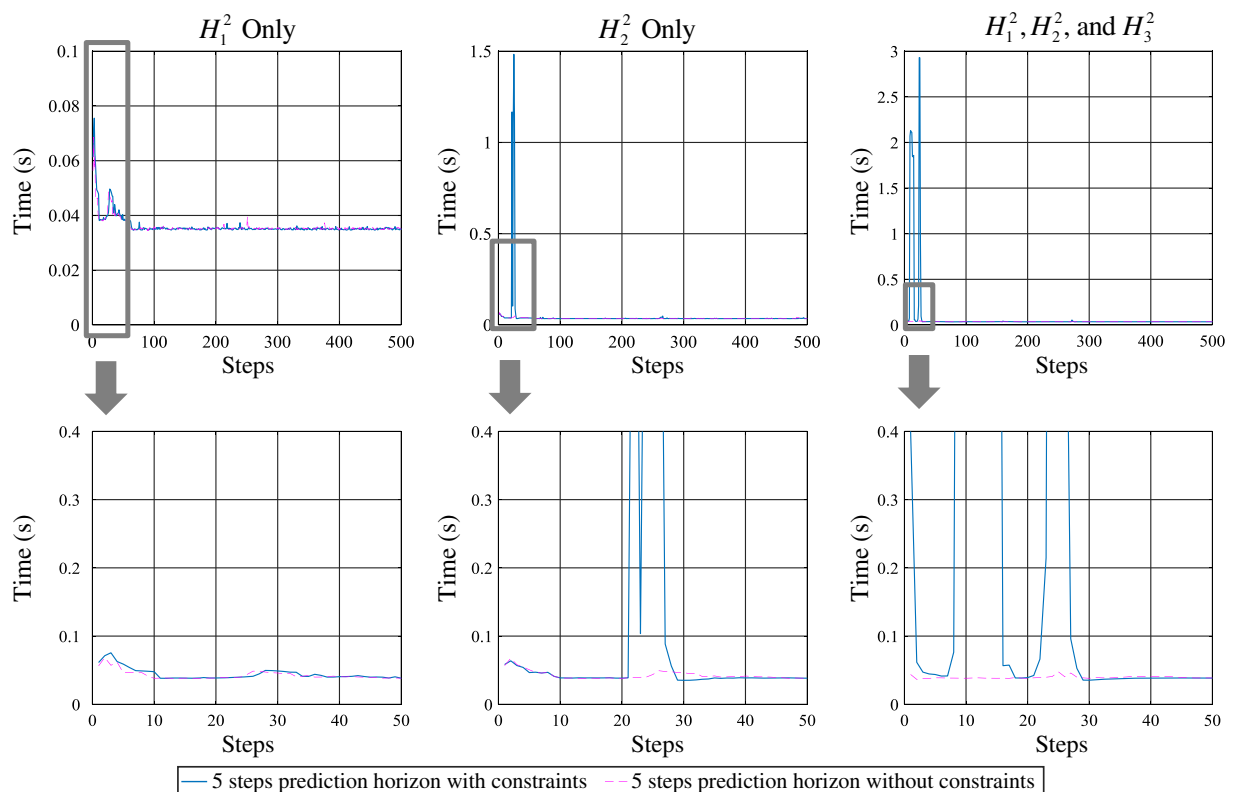

Fig. 6 Computation time comparison for a four-RWA spacecraft on an Intel $^{\circledR}$ i-7 $7^{\circledR}, 3.6$ GHz, 16 GB RAM machine, with MATLAB ${ }^{\circledR}$ 
2) For pitch, $-90,-80,-70, \cdots, 80,90$ deg (19 cases).

3 ) For yaw, 0, 10, 20, $\cdots, 350,360$ deg (37 cases).

The domain of attraction of the controller in [22] has narrower yaw and pitch ranges as compared to the proposed controller. For example, the pitch range larger than \pm 60 deg and yaw range from 90 to $270 \mathrm{deg}$ are not included in the domain of attraction of the controller in [22].

\section{Computation Time}

In Fig. 6, we report the computation times corresponding to three different simulations corresponding to the four-RWA configuration. The first column of Fig. 6 contains the computation time results with only the actuator maximum torque constraint $H_{1}^{2}$ included, the second column contains the same results with only the zone constraint $H_{2}^{2}$ included, and the third column contains the same results with all constraints from the four-RWA example. In the first case, the numerical solver obtains the solution well within the sample time; however, the addition of state constraints makes the solution more difficult to obtain. These results were obtained on MATLAB within a desktop computer environment. We expect that, by converting the proposed algorithm to a $\mathrm{C}$ code, it is possible to speed up the computation by up to about forty times. Yet, this would likely be mitigated by the lower amount of computational power available on an embedded processor. Therefore, to be implemented in real time, the proposed algorithm will benefit from further computational enhancements.

\section{Conclusions}

In this paper, an NMPC approach to the constrained attitude control of spacecraft is presented. The work extends the solver of [20] to the case of spacecraft with a RWA. The work is distinguished by exploiting discrete-time LGVI models that respect the underlying Lie group structure of $\mathrm{SO}(3)$ and are consistent with the geometric mechanics and control formalism. The numerical solution to the NMPC optimization problem is obtained using necessary conditions for optimality and a single shooting method to solve the resulting two-point boundary-value problem. The sensitivity derivatives are used in the numerical solver. The simulation results show that this approach is able to achieve well-behaved closed-loop responses with a large domain of attraction while satisfying a variety of constraints.

\section{Appendix: Derivation of the Necessary Conditions for Optimality of the Spacecraft with RWA}

The variations of $R_{k}, F_{k}, \Pi_{k}$, and $\pi_{k}$ are given as follows:

$$
\begin{aligned}
R_{k, \varepsilon} & =R_{k} \exp \left(\varepsilon \zeta_{k}^{\times}\right) \\
F_{k, \varepsilon} & =F_{k} \exp \left(\varepsilon \zeta_{k}^{\times}\right) \\
\Pi_{k, \varepsilon} & =\Pi_{k}+\varepsilon \delta \Pi_{k} \\
\pi_{k, \varepsilon} & =\pi_{k}+\varepsilon \delta \pi_{k}
\end{aligned}
$$

where $\zeta_{k}, \xi_{k} \in \mathbb{R}^{3}$. Note that $\zeta_{0}=\xi_{0}=\delta \Pi_{0}=\delta \pi_{0}=0$. The infinitesimal variations of $R_{k}, F_{k}, \Pi_{k}$, and $\pi_{k}$ are given by

$$
\begin{aligned}
& \delta R_{k}=\left.\frac{\mathrm{d}}{\mathrm{d} \varepsilon}\right|_{\varepsilon=0} R_{k, \varepsilon}=R_{k} \zeta_{k}^{\times}, \\
& \delta F_{k}=\left.\frac{\mathrm{d}}{\mathrm{d} \varepsilon}\right|_{\varepsilon=0} F_{k, \varepsilon}=F_{k} \xi_{k}^{\times}, \\
& \delta \Pi_{k}=\left.\frac{\mathrm{d}}{\mathrm{d} \varepsilon}\right|_{\varepsilon=0} \Pi_{k, \varepsilon}=\delta \Pi_{k}, \\
& \delta \pi_{k}=\left.\frac{\mathrm{d}}{\mathrm{d} \varepsilon}\right|_{\varepsilon=0} \pi_{k, \varepsilon}=\delta \pi_{k}
\end{aligned}
$$

A few facts are required to proceed further.

Fact 1 [21]: Note that $\zeta_{k}, \zeta_{k+1}$, and $\xi_{k}$ satisfy

$$
\zeta_{k+1}=F_{k}^{T} \zeta_{k}+\xi_{k}
$$

Proposition 1: Note that $\xi_{k}, \delta \Pi_{k}$, and $\delta \pi_{k}$ satisfy

$$
\xi_{k}=\mathcal{B}_{k} \delta \Pi_{k}+\mathcal{E}_{k} \delta \pi_{k}
$$

where

$$
\begin{aligned}
\mathcal{B}_{k} & =h\left(\begin{array}{c}
\left(\operatorname{tr}\left(F_{k} A_{k}\right) I_{3 \times 3}-F_{k} A_{k}\right) F_{k} \\
-\frac{1}{4}\left(\operatorname{tr}\left(F_{k}\right) I_{3 \times 3}-F_{k}^{T}\right) \mathcal{S}\left(J_{w}\right) \mathcal{S}^{T}\left(\operatorname{tr}\left(F_{k}\right) I_{3 \times 3}-F_{k}^{T}\right)
\end{array}\right)^{-1} \\
\mathcal{E}_{k} & =-\mathcal{B}_{k}\left(\frac{1}{2}\left(\operatorname{tr}\left(F_{k}\right) I_{3 \times 3}-F_{k}^{T}\right) \mathcal{S}\right)
\end{aligned}
$$

Proof: We begin with the following:

$$
\begin{aligned}
h \delta \pi_{i_{k}} & =J_{i}\left(\delta \Delta \theta_{i_{k}}-\frac{1}{2} \operatorname{tr}\left(\delta F_{k} s_{i}^{\times}\right)\right)=J_{i}\left(\delta \Delta \theta_{i_{k}}-\frac{1}{2} \operatorname{tr}\left(F_{k} \xi_{k}^{\times} s_{i}^{\times}\right)\right) \\
& =J_{i}\left(\delta \Delta \theta_{i_{k}}-\frac{1}{2} \operatorname{tr}\left(\xi_{k}^{\times} s_{i}^{\times} F_{k}\right)\right) \\
& =J_{i}\left(\delta \Delta \theta_{i_{k}}-\frac{1}{2} \operatorname{tr}\left(\xi_{k}^{\times}\left(\left(M_{i_{k}}\right)_{S}+\left(M_{i_{k}}\right)_{A}\right)\right)\right) \\
& =J_{i}\left(\delta \Delta \theta_{i_{k}}-\frac{1}{2} \operatorname{tr}\left(\xi_{k}^{\times}\left(M_{i_{k}}\right)_{A}\right)\right)=J_{i}\left(\delta \Delta \theta_{i_{k}}-\frac{1}{2} \operatorname{tr}\left(\xi_{k}^{\times} \gamma_{i_{k}}^{\times}\right)\right) \\
& =J_{i}\left(\delta \Delta \theta_{i_{k}}+\xi_{k}^{T} \gamma_{i_{k}}\right)
\end{aligned}
$$

where

$$
\begin{aligned}
M_{i_{k}} & =s_{i}^{\times} F_{k}=\left(M_{i_{k}}\right)_{S}+\left(M_{i_{k}}\right)_{A} \\
\left(M_{i_{k}}\right)_{A} & =\frac{s_{i}^{\times} F_{k}-\left(s_{i}^{\times} F_{k}\right)^{T}}{2}=\frac{s_{i}^{\times} F_{k}+F_{k}^{T}\left(s_{i}^{\times}\right)}{2}=\gamma_{i_{k}}^{\times} \\
\gamma_{i_{k}} & =\frac{1}{2}\left(\operatorname{tr}\left(F_{k}\right) I_{3 \times 3}-F_{k}\right) s_{i}
\end{aligned}
$$

By using the matrices $\mathcal{S}$ and $J_{w}$, we obtain

$$
h \delta \pi_{k}=J_{w}\left(\delta \Delta \theta_{k}+\frac{1}{2} \mathcal{S}^{T}\left(\operatorname{tr}\left(F_{k}\right) I_{3 \times 3}-F_{k}\right)^{T} \xi_{k}\right)
$$

which leads to

$$
\delta \Delta \theta_{k}=h\left(J_{w}\right)^{-1} \delta \pi_{k}-\frac{1}{2} \mathcal{S}^{T}\left(\operatorname{tr}\left(F_{k}\right) I_{3 \times 3}-F_{k}\right)^{T} \xi_{k}
$$

Note that

$$
h\left(\delta \Pi_{k}\right)^{\times}=\delta F_{k} A_{k}-A_{k}^{T} \delta F_{k}^{T}+F_{k} \delta A_{k}-\delta A_{k}^{T} F_{k}^{T}
$$

Using the following properties,

$$
\begin{aligned}
\delta A_{k} & =\frac{1}{2} \sum_{i=1}^{n} s_{i}^{\times}\left(J_{i} \delta \Delta \theta_{i_{k}}\right), \\
F_{k} \delta A_{k}-\delta A_{k}^{T} F_{k}^{T} & =\left(\frac{1}{2} \sum_{i=1}^{n} s_{i}^{\times}\left(J_{i} \delta \Delta \theta_{i_{k}}\right)\right) F_{k}^{T} \\
& +F_{k}\left(\frac{1}{2} \sum_{i=1}^{n} s_{i}^{\times}\left(J_{i} \delta \Delta \theta_{i_{k}}\right)\right) \\
& =\left(\left(\operatorname{tr}\left(F_{k}^{T}\right) I_{3 \times 3}-F_{k}^{T}\right)\left(\frac{1}{2} \sum_{i=1}^{n} s_{i}\left(J_{i} \delta \Delta \theta_{i_{k}}\right)\right)\right)^{\times} \\
& =\left(\frac{1}{2}\left(\operatorname{tr}\left(F_{k}^{T}\right) I_{3 \times 3}-F_{k}^{T}\right) \mathcal{S} J_{w} \delta \Delta \theta_{k}\right)^{\times}
\end{aligned}
$$


we can rewrite Eq. (1ㅡ) as follows:

$$
\begin{aligned}
h\left(\delta \Pi_{k}\right)^{\times} & =F_{k} \xi_{k}^{\times} A_{k}-A_{k}^{T} \xi_{k}^{\times T} F_{k}^{T}+F_{k} \delta A_{k}-\delta A_{k}^{T} F_{k}^{T} \\
& =F_{k} \xi_{k}^{\times} A_{k}+A_{k}^{T} \xi_{k}^{\times} F_{k}^{T}+\left(\frac{1}{2}\left(\operatorname{tr}\left(F_{k}^{T}\right) I_{3 \times 3}-F_{k}^{T}\right) \mathcal{S} J_{w} \delta \Delta \theta_{k}\right)^{\times} \\
& =\left(F_{k} \xi_{k}\right)^{\times} F_{k} A_{k}+A_{k}^{T} F_{k}^{T}\left(F_{k} \xi_{k}\right)^{\times} \\
& +\left(\frac{1}{2}\left(\operatorname{tr}\left(F_{k}^{T}\right) I_{3 \times 3}-F_{k}^{T}\right) \mathcal{S} J_{w} \delta \Delta \theta_{k}\right)^{\times} \\
& =\left(\left(\operatorname{tr}\left(F_{k} A_{k}\right) I_{3 \times 3}-F_{k} A_{k}\right) F_{k} \xi_{k}\right. \\
& \left.+\frac{1}{2}\left(\operatorname{tr}\left(F_{k}^{T}\right) I_{3 \times 3}-F_{k}^{T}\right) \mathcal{S} J_{w} \delta \Delta \theta_{k}\right)^{\times}
\end{aligned}
$$

Applying the inverse of the cross map to both sides of the preceding equation, we obtain

$$
\begin{aligned}
\delta \Pi_{k}= & \frac{1}{h}\left(\operatorname{tr}\left(F_{k} A_{k}\right) I_{3 \times 3}-F_{k} A_{k}\right) F_{k} \xi_{k} \\
& +\frac{1}{2 h}\left(\operatorname{tr}\left(F_{k}^{T}\right) I_{3 \times 3}-F_{k}^{T}\right) \mathcal{S} J_{w} \delta \Delta \theta_{k} \\
= & \frac{1}{h}\left(\operatorname{tr}\left(F_{k} A_{k}\right) I_{3 \times 3}-F_{k} A_{k}\right) F_{k} \xi_{k}+\frac{1}{2 h}\left(\operatorname{tr}\left(F_{k}^{T}\right) I_{3 \times 3}-F_{k}^{T}\right) \\
& \times \mathcal{S}\left(h \delta \pi_{k}-\frac{1}{2} J_{w} \mathcal{S}^{T}\left(\operatorname{tr}\left(F_{k}\right) I_{3 \times 3}-F_{k}\right)^{T} \xi_{k}\right) \\
= & \frac{1}{h}\left(\left(\operatorname{tr}\left(F_{k} A_{k}\right) I_{3 \times 3}-F_{k} A_{k}\right) F_{k}\right. \\
& \left.-\frac{1}{4}\left(\operatorname{tr}\left(F_{k}\right) I_{3 \times 3}-F_{k}^{T}\right) \mathcal{S}\left(J_{w}\right) \mathcal{S}^{T}\left(\operatorname{tr}\left(F_{k}\right) I_{3 \times 3}-F_{k}^{T}\right)\right) \xi_{k} \\
& +\frac{1}{2}\left(\operatorname{tr}\left(F_{k}\right) I_{3 \times 3}-F_{k}^{T}\right) \mathcal{S} \delta \pi_{k}
\end{aligned}
$$

The result follows from the expressions for $\delta \Pi_{k}$ and $\delta \pi_{k}$. $\square$ Fact 2 [21]: Note that $\delta M_{k}=\mathcal{M}_{k} \zeta_{k}$, where $\mathcal{M}_{k} \in \mathbb{R}^{3 \times 3}$. Fact 3 [20]: Note that $\left\langle\left\langle D_{R_{k}} \mathcal{F}, R_{k} \zeta_{k}^{\times}\right\rangle\right\rangle=\left\langle\left(\left(R_{k}^{T}\left(D_{R_{k}} \mathcal{F}\right)\right)_{A}\right)^{-\times}, \zeta_{k}\right\rangle$.

With the preceding facts, the variation of the augmented cost functional can be written as follows:

$$
\begin{aligned}
\delta \mathcal{J}_{d, a}^{2}=\left\langle\left(\left(R_{N}^{T}\left(D_{R_{N}} K_{d}^{2}\right)\right)_{A}\right)^{-\times}, \zeta_{N}\right\rangle+\left\langle D_{\Pi_{N}} K_{d}^{2}, \delta \Pi_{N}\right\rangle+\left\langle D_{\pi_{N}} K_{d}^{2}, \delta \pi_{N}\right\rangle \\
+\sum_{k=1}^{N-1}\left\langle\left(\left(R_{k}^{T}\left(D_{R_{k}} C_{d}^{2}\right)\right)_{A}\right)^{-\times}, \zeta_{k}\right\rangle+\sum_{k=1}^{N-1}\left\langle D_{\Pi_{k}} C_{d}^{2}, \delta \Pi_{k}\right\rangle+\sum_{k=1}^{N-1}\left\langle D_{\pi_{k}} C_{d}^{2}, \delta \pi_{k}\right\rangle+\sum_{k=1}^{N-1}\left\langle D_{\tau_{k}} C_{d}^{2}, \delta \tau_{k}\right\rangle+\sum_{k=0}^{N-1}\left\langle\lambda_{k}^{1},\left(\zeta_{k+1}-F_{k}^{T} \zeta_{k}-\xi_{k}\right)\right\rangle \\
+\sum_{k=0}^{N-1}\left\langle\lambda_{k}^{2},\left(\delta \Pi_{k+1}-\left(F_{k} \xi_{k}^{\times}\right)^{T} \Pi_{k}-F_{k}^{T} \delta \Pi_{k}-h \mathcal{M}_{k} \zeta_{k}\right)\right\rangle+\sum_{k=0}^{N-1}\left\langle\lambda_{k}^{3},\left(\delta \pi_{k+1}-\delta \pi_{k}-h \delta \tau_{k}\right)\right\rangle \\
+\sum_{k=0}^{N-1}\left\langle\sum_{\ell=1}^{m} \mu_{\ell}\left(\left(R_{k}^{T}\left(D_{R_{k}}\left(\phi_{\ell} \circ H_{\ell}^{2}\right)\right)\right)_{A}\right)^{-\times}, \zeta_{k}\right\rangle+\sum_{k=0}^{N-1}\left\langle\sum_{\ell=1}^{m} \mu_{\ell} D_{\Pi_{k}}\left(\phi_{\ell} \circ H_{\ell}^{2}\right), \delta \Pi_{k}\right\rangle \\
+\sum_{k=0}^{N-1}\left\langle\sum_{\ell=1}^{m} \mu_{\ell} D_{\pi_{k}}\left(\phi_{\ell} \circ H_{\ell}^{2}\right), \delta \pi_{k}\right\rangle+\sum_{k=0}^{N-1}\left\langle\sum_{\ell=1}^{m} \mu_{\ell} D_{\tau_{k}}\left(\phi_{\ell} \circ H_{\ell}^{2}\right), \delta \tau_{k}\right\rangle=\left\langle\left(\left(R_{N}^{T}\left(D_{R_{N}} K_{d}^{2}\right)\right)_{A}\right)^{-\times}, \zeta_{N}\right\rangle+\left\langle D_{\Pi_{N}} K_{d}^{2}, \delta \Pi_{N}\right\rangle+\left\langle D_{\pi_{N}} K_{d}^{2}, \delta \pi_{N}\right\rangle \\
+\sum_{k=1}^{N-1}\left\langle\left(\left(R_{k}^{T}\left(D_{R_{k}} C_{d}^{2}\right)\right)_{A}\right)^{-\times}, \zeta_{k}\right\rangle+\sum_{k=1}^{N-1}\left\langle D_{\Pi_{k}} C_{d}^{2}, \delta \Pi_{k}\right\rangle+\sum_{k=1}^{N-1}\left\langle D_{\pi_{k}} C_{d}^{2}, \delta \pi_{k}\right\rangle+\sum_{k=1}^{N-1}\left\langle D_{\tau_{k}} C_{d}^{2}, \delta \tau_{k}\right\rangle+\sum_{k=0}^{N-1}\left\langle\lambda_{k}^{1}, \zeta_{k+1}-F_{k}^{T} \zeta_{k}-\left(\mathcal{B}_{k} \delta \Pi_{k}+\mathcal{E}_{k} \delta \pi_{k}\right)\right\rangle \\
+\sum_{k=0}^{N-1}\left\langle\lambda_{k}^{2},\left(\delta \Pi_{k+1}-\left(F_{k}^{T} \Pi_{k}\right)^{\times}\left(\mathcal{B}_{k} \delta \Pi_{k}+\mathcal{E}_{k} \delta \pi_{k}\right)-F_{k}^{T} \delta \Pi_{k}-h \mathcal{M}_{k} \zeta_{k}\right)\right\rangle+\sum_{k=0}^{N-1}\left\langle\lambda_{k}^{3}, \delta \pi_{k+1}-\delta \pi_{k}-h \delta \tau_{k+1}\right\rangle \\
+\sum_{k=0}^{N-1}\left\langle\sum_{\ell=1}^{m} \mu_{\ell}\left(\left(R_{k}^{T}\left(D_{R_{k}}\left(\phi_{\ell} \circ H_{\ell}^{2}\right)\right)\right)_{A}\right)^{-\times}, \zeta_{k}\right\rangle+\sum_{k=0}^{N-1}\left\langle\sum_{\ell=1}^{m} \mu_{\ell} D_{\Pi_{k}}\left(\phi_{\ell} \circ H_{\ell}^{2}\right), \delta \Pi_{k}\right\rangle \\
+\sum_{k=0}^{N-1}\left\langle\sum_{\ell=1}^{m} \mu_{\ell} D_{\pi_{k}}\left(\phi_{\ell} \circ H_{\ell}^{2}\right), \delta \pi_{k}\right\rangle+\sum_{k=0}^{N-1}\left\langle\sum_{\ell=1}^{m} \mu_{\ell} D_{\tau_{k}}\left(\phi_{\ell} \circ H_{\ell}^{2}\right), \delta \tau_{k}\right\rangle
\end{aligned}
$$

Then,

$$
\begin{aligned}
& \delta \mathcal{J}_{d, a}^{2}=\left\langle\left(\left(R_{N}^{T}\left(D_{R_{N}} K_{d}^{2}\right)\right)_{A}\right)^{-\times}+\lambda_{N-1}^{1}, \zeta_{N}\right\rangle+\left\langle D_{\Pi_{N}} K_{d}^{2}+\lambda_{N-1}^{2}, \delta \Pi_{N}\right\rangle+\left\langle D_{\pi_{N}} K_{d}^{2}+\lambda_{N-1}^{3}, \delta \pi_{N}\right\rangle \\
& +\sum_{k=1}^{N-1}\left\langle\left(\begin{array}{c}
\lambda_{k-1}^{1}-F_{k} \lambda_{k}^{1}-h \mathcal{M}_{k}^{T} \lambda_{k}^{2} \\
+\left(\left(R_{k}^{T}\left(D_{R_{k}} C_{d}^{2}\right)\right)_{A}\right)^{-\times}+\sum_{\ell=1}^{m} \mu_{\ell}\left(\left(R_{k}^{T}\left(D_{R_{k}}\left(\phi_{\ell} \circ H_{\ell}^{2}\right)\right)\right)_{A}\right)^{-\times}
\end{array}\right), \zeta_{k}\right\rangle \\
& +\sum_{k=1}^{N-1}\left\langle\left(\lambda_{k-1}^{2}-\mathcal{B}_{k}^{T} \lambda_{k}^{1}-\left(F_{k}-\mathcal{B}_{k}^{T}\left(F_{k}^{T} \Pi_{k}\right)^{\times}\right) \lambda_{k}^{2}+D_{\Pi_{k}} C_{d}^{2}+\sum_{\ell=1}^{m} \mu_{\ell} D_{\Pi_{k}}\left(\phi_{\ell} \circ H_{\ell}^{2}\right)\right), \delta \Pi_{k}\right\rangle \\
& +\sum_{k=1}^{N-1}\left\langle\left(\lambda_{k-1}^{3}-\mathcal{E}_{k}^{T} \lambda_{k}^{1}+\mathcal{E}_{k}^{T}\left(F_{k}^{T} \Pi_{k}\right)^{\times} \lambda_{k}^{2}-\lambda_{k}^{3}+D_{\pi_{k}} C_{d}^{2}+\sum_{\ell=1}^{m} \mu_{\ell} D_{\pi_{k}}\left(\phi_{\ell} \circ H_{\ell}^{2}\right)\right), \delta \pi_{k}\right\rangle \\
& +\sum_{k=1}^{N-1}\left\langle\left(-h \lambda_{k-1}^{3}+D_{\tau_{k}} C_{d}^{2}+\sum_{\ell=1}^{m} \mu_{\ell} D_{\tau_{k}}\left(\phi_{\ell} \circ H_{\ell}^{2}\right)\right), \delta \tau_{k}\right\rangle
\end{aligned}
$$


where the analog of integration by parts in the discrete-time setting is used along with the facts that the variations $\zeta_{k}, \delta \Pi_{k}$ and $\delta \pi_{k}$ vanish at $k=0$. Because $\delta \mathcal{J}_{d, a}^{2}=0$ should vanish for all variations of $\zeta_{k}, \delta \Pi_{k}$ $\delta \pi_{k}$, and $\delta \tau_{k}$, we have completed the derivation of the necessary conditions given in Eq. (10).

\section{Acknowledgments}

The research of Anthony M. Bloch was supported by National Science Foundation (NSF) grants DMS-1207693, DMS-1613819, INSPIRE-1343720; and the Simons Foundation. The research of Ilya V. Kolmanovsky was supported by NSF grants CNS-1544844 and ECCS-1404814. This work was not sponsored by Mitsubishi Electric Company or any of its subsidiaries.

\section{References}

-11] Mayne, D., Rawlings, J., Rao, C., and Scokaert, P., "Constrained Model Predictive Control: Stability and Optimality," Automatica, Vol. 36, No. 6, 2000, pp. 789-814.

doi:10.1016/S0005-1098(99)00214-9

-[2] Hegrenæs, Ø., Gravdahl, J. T., and Tøndel, P., "Spacecraft Attitude Control Using Explicit Model Predictive Control," Automatica, Vol. 41, No. 12, 2005, pp. 2107-2114. doi:10.1016/j.automatica.2005.06.015

[3] Silani, E., and Lovera, M., "Magnetic Spacecraft Attitude Control: A Survey and Some New Results," Control Engineering Practice, Vol. 13, No. 3, 2005, pp. 357-371. doi:10.1016/j.conengprac.2003.12.017

[4] Vandersteen, J., Diehl, M., Aerts, C., and Swevers, J., "Spacecraft Attitude Estimation and Sensor Calibration Using Moving Horizon Estimation," Journal of Guidance, Control, and Dynamics, Vol. 36, No. 3, 2013, pp. 734-742. doi: $10.2514 / 1.58805$

[5] Guiggiani, A., Kolmanovsky, I., Patrinos, P., and Bemporad, A., "FixedPoint Constrained Model Predictive Control of Spacecraft Attitude," Proceedings of 2015 American Control Conference, IEEE Publ., Piscataway, NJ, July 2015, pp. 2317-2322. doi:10.1109/ACC.2015.7171078

[6] Guiggiani, A., Kolmanovsky, I. V., Patrinos, P., and Bemporad, A., "Constrained Model Predictive Control of Spacecraft Attitude with Reaction Wheels Desaturation," Proceedings of 2015 European Control Conference, IEEE Publ., Piscataway, NJ, 2015, pp. 1376-1381. doi:10.1109/ECC.2015.7330731

- [7] Springmann, J. C., and Cutler, J. W., "On-Orbit Calibration of Photodiodes for Attitude Determination," Journal of Guidance, Control, and Dynamics, Vol. 37, No. 6, 2014, pp. 1808-1823. doi:10.2514/1.G000175

-[8] Wie, B., and Lu, J., "Feedback Control Logic for Spacecraft Eigenaxis Rotations Under Slew Rate and Control Constraints," Journal of Guidance, Control, and Dynamics, Vol. 18, No. 6, 1995, pp. 1372-1379. doi: $10.2514 / 3.21555$

[9] Bang, H., Tahk, M.-J., and Choi, H.-D., "Large Angle Attitude Control of Spacecraft with Actuator Saturation," Control Engineering Practice, Vol. 11, No. 9, 2003, pp. 989-997. doi:10.1016/S0967-0661(02)00216-2

[10] Ali, I., Radice, G., and Kim, J., "Backstepping Control Design with Actuator Torque Bound for Spacecraft Attitude Maneuver," Journal of Guidance, Control, and Dynamics, Vol. 33, No. 1, 2010, pp. 254-259. doi:10.2514/1.45541

[11] Verbin, D., and Lappas, V. J., "Rapid Rotational Maneuvering of Rigid Satellites with Reaction Wheels," Journal of Guidance, Control, and Dynamics, Vol. 36, No. 5, 2013, pp. 1538-1544. doi: $10.2514 / 1.58155$

[12] McInnes, C. R., "Large Angle Slew Maneuvers with Autonomous Sun Vector Avoidance," Journal of Guidance, Control, and Dynamics, Vol. 17, No. 4, 1994, pp. 875-877.

doi: $10.2514 / 3.21283$
[13] Hablani, H. B., "Attitude Commands Avoiding Bright Objects and Maintaining Communication with Ground Station," Journal of Guidance, Control, and Dynamics, Vol. 22, No. 6, 1999, pp. 759-767. doi: $10.2514 / 2.4469$

-[14] Kim, Y., and Mesbahi, M., "Quadratically Constrained Attitude Control via Semidefinite Programming," IEEE Transactions on Automatic Control, Vol. 49, No. 5, May 2004, pp. 731-735. doi:10.1109/TAC.2004.825959

[15] Koenig, J., "A Novel Attitude Guidance Algorithm for Exclusion Zone Avoidance," 2009 IEEE Aerospace Conference, IEEE Publ., Piscataway, NJ, March 2009, pp. 1-10.

[16] Hutao, C., Xiaojun, C., Rui, X., and Pingyuan, C., "RHC-Based Attitude Control of Spacecraft Under Geometric Constraints," Aircraft Engineering and Aerospace Technology, Vol. 83, No. 5, 2011, pp. 296-305.

[17] Kjellberg, H. C., and Lightsey, E. G., "Discretized Constrained Attitude Pathfinding and Control for Satellites," Journal of Guidance, Control, and Dynamics, Vol. 36, No. 5, 2013, pp. 1301-1309. doi:10.2514/1.60189

[18] Kalabić, U., Gupta, R., Di Cairano, S., Bloch, A., and Kolmanovsky, I., "Constrained Spacecraft Attitude Control on SO(3) Using Reference Governors and Nonlinear Model Predictive Control," Proceedings of 2014 American Control Conference, IEEE Publ., Piscataway, NJ, June 2014, pp. 5586-5593. doi:10.1109/ACC.2014.6858865

[19] Kalabić, U. V., Gupta, R., Di Cairano, S., Bloch, A. M., and Kolmanovsky, I. V., "MPC on Manifolds with Applications to the Control of Spacecraft Attitude on SO(3), Automatica," Automatica, 2016.

doi:10.1016/j.automatica.2016.10.022

-[20] Gupta, R., Kalabić, U. V., Di Cairano, S., Bloch, A. M., and Kolmanovsky, I. V., "Constrained Spacecraft Attitude Control on SO (3) Using Fast Nonlinear Model Predictive Control," Proceedings of 2015 American Control Conference, IEEE Publ., Piscataway, NJ, July 2015, pp. 2980-2986. doi:10.1109/ACC.2015.7171188

[21] Lee, T., Leok, M., and McClamroch, N. H., "Optimal Attitude Control of a Rigid Body Using Geometrically Exact Computations on $\mathrm{SO}(3)$," Journal of Dynamical and Control Systems, Vol. 14, No. 4, 2008, pp. $465-487$. doi:10.1007/s10883-008-9047-7

[22] Guiggiani, A., "Embedded Model Predictive Control: Finite Precision Arithmetic and Aerospace Applications," Ph.D. Thesis, IMT Inst. for Advanced Studies, Lucca, Italy, 2015.

[23] Lee, D. Y., Gupta, R., Kalabić, U. V., Cairano, S. D., Bloch, A. M., Cutler, J. W., and Kolmanovsky, I. V., "Constrained Attitude Maneuvering of a Spacecraft with Reaction Wheel Assembly by Nonlinear Model Predictive Control," Proceedings of 2016 American Control Conference, IEEE Publ., Piscataway, NJ, July 2016, pp. 4960-4965. doi:10.1109/ACC.2016.7526139

[24] Kalabić, U., Gupta, R., Cairano, S. D., Bloch, A., and Kolmanovsky, I., "MPC on Manifolds with an Application to SE(3)," Proceedings of 2016 American Control Conference, IEEE Publ., Piscataway, NJ, July 2016, pp. 7-12. doi:10.1109/ACC.2016.7524883

[25] Lee, T., "Computational Geometric Mechanics and Control of Rigid Bodies," Ph.D. Thesis, Univ. of Michigan, Ann Arbor, MI, 2008.

[26] Lee, T., and Leve, F., "Lagrangian Mechanics and Lie Group Variational Integrators for Spacecraft with Imbalanced Reaction Wheels," Proceedings of 2014 American Control Conference, IEEE Publ., Piscataway, NJ, June 2014, pp. 3122-3127. doi:10.1109/ACC.2014.6859086

[27] Ismail, Z., and Varatharajoo, R., "A Study of Reaction Wheel Configurations for a 3-Axis Satellite Attitude Control," Advances in Space Research, Vol. 45, No. 6, 2010, pp. 750-759. doi:10.1016/j.asr.2009.11.004

[28] Fletcher, R., Practical Methods of Optimization, Wiley, Hoboken, NJ, 2013, pp. 296-304. 